\title{
Kennen Sie Morgellonen?
}

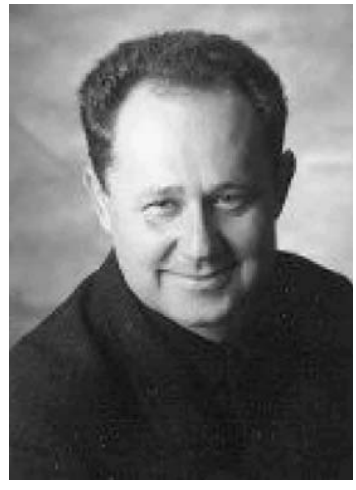

Prof. Dr. Manfred Wildner
Bibliografie

DOI http://dx.doi.org/ 10.1055/s-0029-1242727

Gesundheitswesen 2009;

71: 795-796

(c) Georg Thieme Verlag KG

Stuttgart · New York

ISSN 0941-3790

Korrespondenzadresse

Prof. Dr. M. Wildner

Bayerisches Landesamt für

Gesundheit und Lebens-

mittelsicherheit

Veterinärstraße 2

85762 Oberschleißheim

manfred.wildner@Igl.bayern.de
Dienstag, 10. Februar. Bürger an Pressestelle: „Seit letztem Jahr bin ich an der sog. MorgellonsDisease erkrankt. Diese äußert sich in Hautläsionen, Abgeschlagenheit, Muskelschmerzen usw. Die CDC in den USA sind bereits an der Erforschung dieser Krankheit maßgeblich involviert. Auf der Internetseite http://www.morgellons.org können Sie nachlesen, dass höchstwahrscheinlich ein $\mathrm{Zu}$ sammenhang mit kontaminiertem Grundwasser besteht. Ich habe daraufhin unser Wasser aus Bad und Küche mit dem Mikroskop untersucht. Dabei stellte ich Verunreinigungen fest, die den Pathogenen der Morgellons Erkrankung verblüffend ähnlich sehen“. Dienstag, 10. Februar. Pressestelle an Fachabteilung: „Anbei eine Verbraucheranfrage mit der Bitte um weitergehende Bearbeitung Mittwoch, 11. Februar. Fachabteilung an Pressestelle: „Dr. B., unser Experte für Morgellonen, wird sich um eine Antwort kümmern. Kurzes Zitat aus „EsoWatch“ [Internetseite mit kritischen Evaluationen esoterischer Thesen, URL: www.esowatch. com]: ,Der Begriff Morgellonen (engl. morgellons) bezeichnet eine geheimnisvoll erscheinende, bizarre und angeblich neuartige Hauterkrankung, die teilweise in den Medien Beachtung fand und als eine Krankheitserfindung (disease mongering) angesehen werden kann. Alle Anzeichen sprechen dafür, dass es sich um eine seit mehreren Jahrhunderten bekannte psychiatrische Erkrankung handelt, die als Dermatozoenwahn oder Ekbom-Syndrom bezeichnet wird und auch bei Hypochondern beobachtet wird [1]: In diesem Sinne viele Grüsse“!

Wir wissen nicht, was aus diesem Patienten geworden ist. Patienten? Mancher Leser wird dieser Bezeichnung durchaus zustimmen, die Zuständigkeit jedoch weniger bei den Experten für Krankheiten der Haut und Hautanhangsgebilde sehen als vielmehr bei den für die psychischen Funktionen des ganzen Menschen zuständigen Kollegen. Ist damit die Akte geschlossen? Individualmedizinisch vielleicht, bevölkerungsmedizinisch sicher nicht. Mögliche Herangehensweisen an derartige Phänomene sind vielfältig: lesenswert aus dem Bereich der Anthropologie/ Ethnomedizin ist der hintergründige Aufsatz über den nordamerikanischen Stammes der Nacirema. Diese teilen den Glauben, dass der menschliche Körper im Grunde hässlich sei und anfällig für Schwäche und Krankheit. Die geheimnisvollen Körperrituale dieses Volkes mit entwickelter Marktwirtschaft kreisen daher um Gesundheit und Aussehen. Übrigens: Nacirema steht - rückwärts gelesen - für Amerikaner [2]. Verblüffend sind die Beobachtungen über Mass Sociogenic Illness (MSI) als hysterisches Grup- penphänomen mit klinisch objektivierbaren Symptomen [3]. Ein Beispiel: reale Atemwegssymptome wurden von $40 \%$ der vermeintlich exponierten israelischen Zivilbevölkerung nach Raketenangriffen während des Golfkrieges angegeben, bei denen fälschlich Giftgas-Sprengköpfe vermutet wurden. Alters- und Geschlechts (rollen) effekte für das Auftreten somatoformer Symptome und hypochondrischer Merkmale sind für die deutsche Allgemeinbevölkerung beschrieben. Diese treten in jüngeren Jahren bei Frauen doppelt so häufig auf und gleichen sich zwischen den Geschlechtern ab dem 45. Lebensjahr mit einer Häufigkeit von 15\% und mehr an [4].

All das ein Laienphänomen? Bei weitem nicht: Die Gesundheitswissenschaftler Diethelm und McKee wiesen auf Strategien der systematischen Verwirrung von Gedanken in Zusammenhang mit skrupellosen Machenschaften der Tabakindustrie hin - und nannten dieses Phänomen „Denialism“ [5]. Unterschieden werden dabei fünf charakteristische Elemente: 1. die Vermutung einer Verschwörung, 2. das Auftreten falscher „Experten“ und die Marginalisierung echter Experten, 3. die selektive Berücksichtigung von Evidenzbruchstücken, 4. die überzogene Erwartungshaltung an das, was Wissenschaft leisten kann und 5. der kontextirrelevante Gebrauch von Fakten und offene logische Fehlschlüsse. Ein erhellendes Lehrstück dazu ist die Debatte zwischen Kuhn und Ropohl [6]. In engem Zusammenhang damit stehen Phänomene des „Pseudowissenschaftlichen“" [7].

Doch nicht nur die Verleugnung von Krankheit bzw. Krankheitsrisiken hat Konjunktur, sondern insbesondere und in einem sehr wörtlichen Sinne verstanden auch ihre Erzeugung. Schon früh haben der französische Philosoph Michel Foucault [8] und der österreichisch-amerikanische Philosoph und katholische Priester Ivan Illich auf die soziale Produktion von Krankheitsvorstellungen hingewiesen und in diesem Zusammenhang z.B. Medikalisierungstendenzen natürlicher Lebensvorgänge wie der Geburt kritisiert. Illich bezieht sich in seiner „Nemesis der Medizin“ [9] auf die griechische Mythologie: Nemesis ist die Göttin des „gerechten Zorns“ und bestraft vor allem die menschliche Selbstüberschätzung (Hybris) und die Missachtung des Rechts und der Sittlichkeit. Kein Problem in Deutschland? Das Statistische Bundesamt teilt in seiner Pressemitteilung vom 07.02.2007 (Nr. 050) mit, dass der Anteil der Entbindungen durch Kaiserschnitt zwischen 1995 und 2005 von jeder fünften Geburt auf fast jede dritte Geburt angestiegen ist: 183000 von etwa 664000 Geburten. Ein implausibler Anstieg al- 
lein auf Basis Risiko-bezogener Indikationsstellung. In welcher Form veränderte Praxisstile, angebotsinduzierte Nachfragen, Patientinnenwünsche und soziale Körperbilder hierbei zusammenspielen, wäre eine eigene Fragestellung. „Implausible Erkrankungen“ wie Colon irritabile, soziale Phobie, Restless Leg Syndrome und männliche Kahlköpfigkeit hat auch der Schweizer Gesundheitswissenschaftler Fred Paccaud in einem Editorial aufgegriffen [10]. Er zitiert Payers Begriff der „Krankmacher“: Gemeint ist eine „Strategie, die darauf abzielt, die Grenzen des Behandelbaren auszuweiten, um die Märkte für all diejenigen zu erweitern, die Therapien anbieten bzw. daran verdienen“ [11]. Moynihan und Mitarbeiter haben insbesondere das Aktionsmuster der pharamzeutischen Industrie unter die Lupe genommen und festgestellt, dass an Stelle der Illich-Foucaultschen sozialen Konstruktion von Krankheit inzwischen die machtvolle Orchestrierung der Medizinischen Versorgungsindustrie getreten ist: „die soziale Konstruktion von Krankheit wurde inzwischen ersetzt durch die Firmen-gesteuerte Konstruktion der Syndrome“ [12].

Sind wir nur gesund, wenn wir krank sind? Weiter ausgeführt wurde diese Kritik von der US-amerikanischen Ärztin Marcia Angell. Bekannt wurde sie für ihr Buch „Der Pharma-Bluff“ [13]. Sie bemängelt Pharma-Forschung gegen Impotenz und Fettleibigkeit an der Grenze zwischen Gesundheit und Krankheit an Stelle von notwendiger Forschung gegen Tropenkrankheiten oder echter Innovation: Das Problem sei das Streben nach Profit an Stelle von Bemühung um die Gesundheit. Solche Vermutungen wurden auch im Zusammenhang mit der Impfstoffbeschaffung gegen die Neue Influenza A/H1N1 („Schweinegrippe“) geäußert. Ob sie tatsächlich zutreffen, sollte im Einzelfall sorgfältig geprüft werden: auch für die „weißen Ritter“ fachlich und ethisch hochstehender Wissenschaft besteht die Gefahr, Verschwörungstheorien zum Opfer zu fallen.

Die wissenschaftliche Gemeinschaft ist sich der Versuchungen des Mammon bewusst und legt strenge Regeln hinsichtlich Interessenkonflikten bei Publikationen vor. Unabdingbar ist eine konsequente Offenlegung, wenn denn solche Konflikte bestehen. Unterschieden wird zwischen materiellen und nichtmateriellen Interessenskonflikten in der Gegenwart und den vergangenen fünf Jahren. Erstere sind beispielsweise Beschäftigungsverhältnisse, Beratungstätigkeiten, Aktienbesitz, Honorare für Vorträge, Reisekostenübernahmen, Studienunterstützung oder andere Drittmittel, letztere betrifft enge persönliche Verbindungen [14].

Von größter Bedeutung sind fehlerfreie, zumindest unbeeinflusste Bewertungen im Zusammenhang mit Gutachten. Hierbei helfen z.B. Leitlinien und eine konsequente Evidenzbasierung. Diese Ausgabe unserer Zeitschrift bemüht sich um beides: Themen der Originalarbeiten sind u.a. Rentenanträge wegen Erwerbsminderung, Diagnosis Related Groups in der Krankenhausfinanzierung, hausarztzentrierte Versorgung und die Validität von Abrechnungsdiagnosen in hausärztlichen Praxen, unspezifische Rückenschmerzen und chronisch-obstruktive Atemwegserkrankungen sowie die Problemlagen von versteckt lebenden Migranten in Deutschland. Hinzu kommt der vertiefende Beitrag zu Lebensqualität des Memorandums zu Methoden der Versorgungsforschung des Deutschen Netzwerks Versorgungsforschung. Von besonderem Interesse für unsere Leser dürfte auch die gemeinsame Empfehlung der AWMF und der DGUV in Zusammenarbeit mit der DGAUM und der DGSMP bei der Entwicklung von Leitlinien und Empfehlungen zur Begutachtung von Berufskrankheiten sein - siehe auch das begleitende Editorial.
A propos Grippeviren(-Verleugnung): noch einmal ein Fallbericht. Bürger an Pressestelle, Freitag 11. September: „Vielen Dank für Ihr Schreiben vom 7. September. Schade, dass Sie mir zu der vom Labor gemachten Aufnahme eines Grippevirus keine näheren Angaben machen können. Können Sie mir eine wissenschaftliche Publikation nennen, in der ein ebensolches Grippevirus fotografiert und beschrieben ist"? Fachabteilung an Bürger, Freitag 11. September: „Vielen Dank für Ihre Anfrage. Eine allgemeinverständliche historische Darstellung der Entdeckung von Influenzaviren einschließlich der betroffenen Quellen finden Sie beispielsweise hier [...] oder hier [...]. Elektronenmikroskopische Darstellungen von Viren finden Sie hier [...]. Bei weitergehendem Interesse würden wir Ihnen empfehlen, in der mehr als umfangreichen wissenschaftlichen Literatur, die Sie beispielsweise in der Datenbank PubMed recherchieren können, noch offene Fragen Ihrerseits $z u$ klären“. Bürger an Fachabteilung, Montag, 21. September: „Vielen Dank für Ihre Email vom 11. September. Die von Ihnen genannten Quellen liefern keine eindeutigen Virusbeweise. Warum wird von Seiten der Medizin so fest an der Virusthese festgehalten? Wenn man ein Virus behaupten kann, dann lassen sich auch leichter Medikamente und Impfungen (mit schweren Nebenwirkungen!!) unters Volk bringen. Gerne höre ich wieder von Ihnen“. Fachabteilung an Bürger, Donnerstag 24. September: „Sehr geehrter Herr S., ich darf Sie auf meine ABSCHLIEßENDE Stellungnahme vom 11.9. verweisen“. „Flame“ nennt sich in der Email-Community die Verwendung von Großbuchstaben bei ganzen Wörtern, im Grundsatz quasi eine elektronische Beleidigung, der erhebliche Lautstärke in der gesprochen Sprache entspricht. Um eine wirklich abschließende Stellungnahme dürfte es sich nach allgemeiner Erfahrung dennoch nicht gehandelt haben.

\section{Literatur}

1 Accordino RE. Morgellons disease? Dermatol Ther 2008 Jan-Feb; 21 (1): $8-12$

2 Miner H. Body ritual among the Nacirema. Am Anthropologist 1956; 58: 503-507 http://web.archive.org/web/20061004083040/http:// www.aaanet.org/pubs/bodyrit.pdf (Zugriff am 16.10.2009)

3 Bartholomew RE, Wessely S. Protean nature of mass sociogenic illness. From possessed nuns to chemical and biological terrorism fears. The British Journal of Psychiatry 2002; 180: 300-306

4 Rief $W$, Hessel A, Braehler E. Somatization symptoms and hypochondrial features in the general population. Psychosomatic Medicine 2001; 63: 595-602

5 Diethelm P, McKee M. Denialism: what it is and how should scientists respond? Eur J Public Health 2009; 19: 2-4

6 Kuhn J. Gesundheitspolitik zwischen Evidenzbasierung und Bürgerorientierung. Prävention 2009 (03): 88-92

7 Rupnow D, Lipphardt V, Thiel J et al., Hrsg. Pseudowissenschaft. Konzeptionen von Nichtwissenschaftlichkeit in der Wissenschaftsgeschichte. Frankfurt a.M., Suhrkamp; 2008

8 Foucault M. Il faut defendre la societe. Cours au College de France, 1967. Paris, Gallimard-Seuil; 1997

9 Illich I. Medical nemesis: the expropriation of health. London, Marian Boyars 1975, [Deutsch: Die Nemesis der Medizin. Von den Grenzen des Gesundheitswesens. Berlin, Rowohlt Verlag, 1982]

10 Paccaud F. Implausible diseases and public health. Eur J Public Health 2007; 17: 410

11 Payer L. Disease mongers. New York, John Wiley; 1992

12 Moynihan R, Heath I, Henry D et al. Selling sickness: the pharmaceutical industry and disease mongering. Commentary: medicalisation of risk factors. Br Med J 2002; 324: 886-891

13 Angell $M$. The truth about the drug companies: how they deceive us and what to do about it. London, Random House; 2004, [Deutsch: Der Pharma-Bluff. Wie innovativ die Pillenindustrie wirklich ist. Berlin, Kompart, 2005]

14 Baethge C. Transparente Texte. Deutsches Ärzteblatt 2008; 105: 675-679 